\title{
Very low birth weight infants receive full enteral nutrition within 2 postnatal weeks
}

\author{
Audrey Fenin ${ }^{1} \cdot$ Jill C. Newman ${ }^{2} \cdot$ Sarah N. Taylor $\mathbb{1}^{3}$
}

Received: 16 January 2020 / Revised: 30 July 2020 / Accepted: 10 September 2020 / Published online: 29 September 2020

(c) The Author(s), under exclusive licence to Springer Nature America, Inc. 2020

\begin{abstract}
Objective Identify whether an enteral nutrition goal of reaching full feeds by 7 postnatal days for infants $1-1.5 \mathrm{~kg}$ and by 14 postnatal days for infants $<1 \mathrm{~kg}$ was feasible and its associated outcomes.

Study design Very low birth weight infant cohort admitted in the first postnatal day and categorized as either Epoch 1 or Epoch 2, 12 months before and after implementation of a revised feeding protocol were compared.

Result In Epoch 2, 83\% infants born 1-1.5 kg and 77\% infants born $<1 \mathrm{~kg}$ reached full feeds by 7 and 14 days compared to $26 \%$ and $25 \%$, respectively in Epoch $1(p<0.0001)$. Central line and parental nutrition days were significantly lower in Epoch 2 compared to Epoch 1 with sustained and potentially improved infant growth.

Conclusion An evidence-based advancement feeding protocol was associated with achieving full feeds within the first 2 postnatal weeks for very low birth weight infants.
\end{abstract}

\section{Introduction}

Debate persists in very low birth weight (VLBW) infant care about the initiation and advancement of enteral nutrition [1-3]. Historically, early introduction to enteral feeding and rapid advancement of feeds were related to higher incidence of necrotizing enterocolitis (NEC) [4-6]. However, current evidence demonstrates no benefit to slower enteral feed advancement $(15-20 \mathrm{~mL} / \mathrm{kg} /$ day compared to $30-40 \mathrm{~mL} / \mathrm{kg} /$ day) and potential harm including a delay in time to achieve full feeds, longer time to regain birth weight, and an increased chance of invasive infection risk with no reduction in the risk of NEC [7, 8]. On the other hand, no difference in NEC or late-onset sepsis was observed in a recently published randomized, controlled

Sarah N. Taylor

sarah.n.taylor@yale.edu

1 Department of Pediatrics, Medical University of South Carolina, 10 McClennan Banks Drive, MSC 915, Charleston, SC 29425, USA

2 Department of Medicine, Medical University of South Carolina, 114 Doughty Street, Charleston, SC 29425, USA

3 Department of Pediatrics, Yale School of Medicine, 333 Cedar Street, New Haven, CT 06510, USA trial [9]. Despite the lack of difference in 2-year outcomes including survival without moderate or severe neurodevelopmental disability in this trial [9], neonatal care centers may still find benefit if more rapid advancement of enteral feeds is associated with two common quality indicators in neonatal care-early discontinuation of central venous lines (CVL) and shorter duration of parenteral nutrition (PN), while maintaining infant growth.

Based on published guidelines recommending advancement of enteral nutrition to achieve full feeds by 7 days in infants born $1-1.5 \mathrm{~kg}$ and by 14 days in infants born $<1 \mathrm{~kg}$ [10], a nutrition-focused research team, whose work to initiate enteral nutrition in the first postnatal day has been published previously [11], aimed to investigate its ability to establish full enteral nutrition $(120 \mathrm{kcal} / \mathrm{kg} /$ day $)$ by 7 postnatal days for infants born $1-1.5 \mathrm{~kg}$ and by 14 postnatal days for infants born $<1 \mathrm{~kg}$ after instituting practices that included discontinuation of routine gastric residual monitoring, decreased days of trophic feeding (minimal enteral nutrition), and faster feed volume advancement. Despite evidence that early total enteral feeding of $50-80 \mathrm{~mL} / \mathrm{kg} /$ day are tolerated on the first postnatal day in infants $1-1.5 \mathrm{~kg}$ [12], concern existed that aggressive early feeding would lead paradoxically to feeding intolerance or fear of feeding intolerance and, therefore, would be associated with a greater delay to achieve full enteral nutrition. Therefore, this cohort study, with a retrospective control, was performed 
with a primary aim to determine if infants were able to reach the full enteral nutrition goal and with secondary aims to determine whether this outcome was associated with changes in PN exposure, CVL days, or growth.

\section{Patients and methods}

After obtaining institutional review board exemption at the Medical University of South Carolina, this retrospective cohort study was performed at a single university-based tertiary care neonatal intensive care unit. Data were retrospectively collected from a nutrition quality improvement database. Subjects were selected if birth weight $\leq 1500 \mathrm{~g}$ and admitted within the first 24 postnatal hours to the neonatal intensive care unit. Infants with congenital anomalies, cardiac defects, or metabolic defects that precluded feeding within the first 24 postnatal hours were excluded as were infants who died in the first 24 postnatal hours, or those discharged home or transferred before 28 postnatal days as their $\mathrm{GV}$ at 28 days would not be calculated. Infants born from March 1, 2017 to February 28, 2018 and meeting inclusion criteria were in the cohort labeled Epoch 1. These dates were chosen as March 1, 2018 was the day of implementation of the new protocol. Infants born March 1, 2018 to February 28, 2019 were included in the Epoch 2 cohort.

Data collected included birthweight, gestational age (GA), sex, race, and ethnicity. In addition, antenatal steroid exposure, small-for-gestational age (SGA) status, respiratory support in the first 28 postnatal days, feeding type, and hour of first feed were collected. SGA was defined as birth weight $<10$ th percentile by Fenton growth reference chart [13]. Feeding type was differentiated as mother's milk (MM) only, MM and donor human milk (DHM) combined, and DHM only. Respiratory support was collected as any oxygen, conventional ventilator, or nasal continuous positive airway pressure (CPAP) in the first 28 postnatal days. Occurrence of sepsis, bronchopulmonary dysplasia (BPD), and NEC was collected through the hospitalization. Sepsis was defined as any culture-positive blood infection during hospitalization. BPD was defined as oxygen support required at 36 weeks' GA or at hospital discharge if prior to 36 weeks' GA. NEC was determined if modified Bell's stage 2 or greater was diagnosed during hospitalization. Spontaneous perforation was determined if occurred in first 28 postnatal days and without a diagnosis of NEC.

CVL and PN number of days and time to full feeds were calculated by finding the difference between the recorded times of initiation and discontinuation for each measure. Growth trajectory was measured as 28-day GV, days to return to birth weight, and the change in weight $z$-score from birth to 28 days as determined by the Fenton growth reference chart [13]. 28 day $\mathrm{GV}$ was chosen as the primary indicator of growth and was calculated by the 2-point model (change in weight in grams from birth to 28 days/the average weight between birth and 28 days/28 days) [14]. Days to return to birth weight was calculated as the first postnatal day at which the infant's weight was higher than the birth weight.

Infants in Epoch 1 had feeds initiated and advanced per a previous feeding protocol adopted in 2010. Infants in Epoch 2 had feed initiation and advancement per the revised protocol instituted on March 1, 2018. The revised feeding protocol was the result of the VLBW infant feeding evidence and expert recommendations published prior to October 31, 2017 as reviewed by an institutional multidisciplinary team comprising neonatologists, nurses, neonatal nurse practitioners, and neonatal registered dietitians. The March 2018 revised protocol included continuation of the practice of initiating feeds at 6-24 postnatal hours, discontinuation of the practice of routine gastric residual monitoring, a decrease in days of trophic feeding of $12 \mathrm{ml} /$ $\mathrm{kg}$ /day from 5 to 3 days in infants $<1 \mathrm{~kg}$ and from 3 to 1 day in infants $1-1.5 \mathrm{~kg}$. The revised protocol also included institution of the practice of faster advancement of enteral feeds with infants $<1 \mathrm{~kg}$ advanced at $25 \mathrm{~mL} / \mathrm{kg} /$ day divided into two daily steps (morning and evening) with fortification to $24 \mathrm{kcal} / \mathrm{oz}$ at $\sim 100 \mathrm{~mL} / \mathrm{kg} / \mathrm{day}$ and infants $1-1.5 \mathrm{~kg}$ advanced at $30 \mathrm{~mL} / \mathrm{kg} /$ day also with two daily steps with fortification at $\sim 110 \mathrm{~mL} / \mathrm{kg} /$ day. In Epoch 1 , infants $<1 \mathrm{~kg}$ advanced at $\sim 18 \mathrm{~mL} / \mathrm{kg} /$ day, also divided in two daily steps with a two-step fortification of $22 \mathrm{kcal} / \mathrm{oz}$ for $12 \mathrm{~h}$ followed by $24 \mathrm{kcal} / \mathrm{oz}$ at $100 \mathrm{~mL} / \mathrm{kg} / \mathrm{day}$. For infants $1-1.5 \mathrm{~kg}$, advancement was also done in two daily steps of $25 \mathrm{~mL} / \mathrm{kg} /$ day, fortified at $100 \mathrm{~mL} / \mathrm{kg} /$ day in two steps also. The practice of introduction of enteral feeds at 6-24 postnatal hours was continued since an earlier study had shown this practice was associated with decreased central line infections, decreased feeding intolerance and improved GV [11]. Infants in both Epoch 1 and 2 were either fed MM or DHM after obtaining parental assent if MM not available. To facilitate institution of the protocol, physicians, nursing, and dietary staff were educated prior to the protocol change by oral and visual presentations. See Fig. 1 for a schematic of the new protocol change. No other changes in nutrition delivery, including PN, occurred during the study period.

\section{Statistical analyses}

Power analysis demonstrated to achieve $90 \%$ power to detect a difference between the group proportions of 0.2 , 119 subjects were needed in each epoch to identify whether the proportion of infants achieving full feeds differ by 0.2 


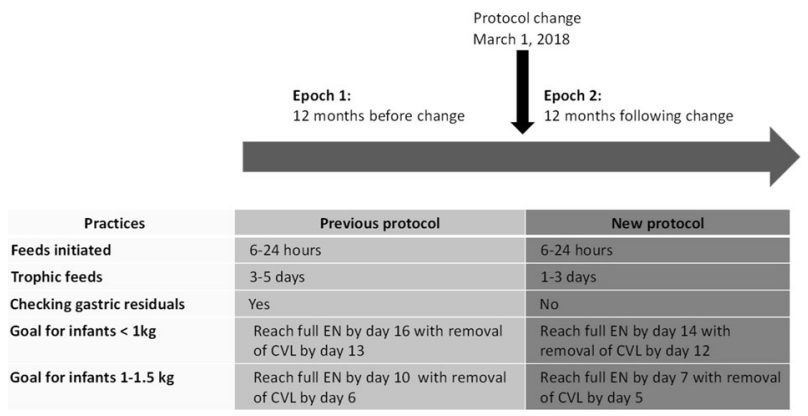

Fig. 1 Schematic of protocol change. ${ }^{*} E N$ enteral nutrition; ${ }^{*} C V L$ central venous line.

from the 0.46 proportion at baseline. A two-sided $Z$ test with pooled variance was used with a targeted significance level at 0.05 . With an estimated 160 eligible subjects born each year, 1-year pre- (Epoch 1) and 1-year post (Epoch 2) were chosen as the ranges for subject inclusion. Descriptive statistics for demographic and outcome characteristics were reported as frequencies and percentages, means and standard deviations or median and interquartile ranges. For the primary aim, a dichotomous (yes/no) variable to indicate whether the infant met the goal for full enteral nutrition was defined. Infants were stratified by birth weight $(<1 \mathrm{~kg}$ and $1-1.5 \mathrm{~kg}$ ). For infants whose birth weight was $<1 \mathrm{~kg}$, the infant met goal if the number of days to full enteral nutrition was less than or equal to 14 postnatal days. If the number of days was $>14$ postnatal days, then the infant did not meet the goal for full enteral nutrition. The same definition applied to birth weight $1-1.5 \mathrm{~kg}$ with the cut-point for number of days being 7 .

Chi-square tests or Fisher's Exact tests were used to test for associations between categorical measures. Since the distributions for number of days CVL and PN were skewed, Wilcoxon Rank Sum Tests were used for unadjusted associations. Student's $t$ test was used to test for associations of normally-distributed continuous measures. Multivariate linear and generalized mixed models were used to assess differences in epoch for GV, number of CVL days, and number of PN days, after controlling for birth GA, birth weight, race/ethnicity, sex, and antenatal steroid exposure. Due to concern for collinearity, further comparisons were performed using SGA status (yes or no) instead of birth weight in all models. Weight $z$-score change from birth to 28-days also was assessed as the dependent variable in this second regression model.

A bar chart was created to show the proportion of infants that met the goal for full enteral nutrition by epoch and weight group. Histograms were used to show the distributions of continuous outcome measures by group. A $p$ value $<0.05$ was considered statistically significant and all analyses were performed using SAS version 9.4 (Cary, NC).

\section{Results}

A total of 272 infants met inclusion criteria. In Epoch 1, 34 subjects were excluded ( 20 due to discharge or transfer to another hospital, 13 due to death, and 1 with congenital anomaly affecting feeding). In Epoch 2, 32 subjects were excluded (14 due to discharge or transfer to another hospital, 14 due to death, and 4 with congenital anomaly affecting feeding).

The two groups were similar in baseline demographics, including SGA status at birth, as shown in Tables 1 and 2. In comparison of feeding exposures which were not affected by the revised feeding guideline, the proportion of infants receiving a combination of DHM feeds, as well as those receiving only DHM were not significantly different between epochs. In addition, both epochs demonstrated initiation of feeds at a median of $9 \mathrm{~h}$. Respiratory exposure in the first 28 postnatal days did not significantly differ between groups except a significantly higher proportion of infants born 1 to $1.5 \mathrm{~kg}$ received nasal CPAP in Epoch 2 .

In Epoch 2, 83\% of infants born 1-1.5 kg achieved full enteral feeds by 7 days and $77 \%$ of infants born $<1 \mathrm{~kg}$ achieved full enteral feeds by 14 days and these proportions were significantly higher than for similar infants in Epoch 1 (Fig. 2). CVL and PN days were also significantly lower in Epoch 2 compared to Epoch 1 (Table 1) and the significant difference remained apparent when stratified by weight group (Table 2). Only 103 infants out of the total 124 infants in Epoch 1 had CVL and 112 out of 148 infants in Epoch 2 had CVL. The distribution of days with PN and CVL between epochs and by weight group is shown in Fig. 3.

In unadjusted analyses, no difference in the mean 28-day $\mathrm{GV}$, weight $z$-score change from birth to 28 postnatal days, or average days to return to birth weight were observed between epoch, even when stratified by weight group (Tables 1 and 2). Multivariate analyses were performed for three secondary outcomes, CVL and PN days and 28-day GV. Initially, regression models were performed with independent variables GA, birth weight, sex, race and ethnicity, and antenatal steroid exposure with significantly lower CVL and PN day in Epoch 2 versus Epoch 1 ( $p$ value $<0.001$ for both comparisons). In addition, in an adjusted model, GV from birth to 28 days was significantly higher in Epoch 2 versus Epoch $1(\beta$ estimate $(\beta)=0.95$, standard error $(\mathrm{SE})=0.35$ and $\mathrm{p}$-value $=0.008)$. Due to concern for collinearity with birth GA and weight in the same model, a second model was developed with SGA versus non-SGA as a covariate instead of birth weight. The results of significantly higher $\mathrm{GV}$ in epoch 2 versus epoch 1 did not differ in the new multivariate model $(\beta=0.85, \mathrm{SE}=0.39$ and $p$ value 0.0286 ). Consistent with previous findings, the number of CVL days remained significantly lower in Epoch 
Table 1 Baseline demographic and outcomes of the study sample overall and by epoch.

\begin{tabular}{|c|c|c|c|c|}
\hline Descriptor & Overall $n=270$ & $\begin{array}{l}\text { Epoch } 1 n= \\
123(45.6)\end{array}$ & $\begin{array}{l}\text { Epoch } 2 n= \\
147(54.4)\end{array}$ & $p$ value* \\
\hline Female $^{\mathrm{a}}$ & $143(53.0 \%)$ & $68(47.6)$ & $75(52.4)$ & 0.48 \\
\hline Race/ethnicity ${ }^{\mathrm{a}}$ & & & & 0.88 \\
\hline Non-Hispanic black & $148(55.6)$ & $66(55.0)$ & $82(56.2)$ & \\
\hline Non-Hispanic white & $105(39.5)$ & $47(39.2)$ & $58(39.7)$ & \\
\hline Hispanic & $10(3.8)$ & $5(4.2)$ & $5(3.4 \%)$ & \\
\hline Other & $3(1.1)$ & $2(1.7)$ & $1(0.7)$ & \\
\hline Antenatal steroids ${ }^{\mathrm{a}}$ & $233(87.6)$ & $104(86.7)$ & $129(88.4)$ & 0.68 \\
\hline $\mathrm{SGA}^{\mathrm{a}}$ & $20(7.5)$ & $10(8.3)$ & $10(6.9)$ & 0.65 \\
\hline Birth weight $(\mathrm{kg})^{\mathrm{b}}$ & $1.0(0.3)$ & $1.02(0.3)$ & $1.05(0.3)$ & 0.34 \\
\hline Birth GA (weeks) ${ }^{b}$ & $28.1(2.4)$ & $28.1(2.6)$ & $28.1(2.2)$ & 0.76 \\
\hline First feed (postnatal hours) ${ }^{\mathrm{c}}$ & $9[7,13]$ & $9[7,16]$ & $9[7,12]$ & 0.34 \\
\hline \multicolumn{5}{|l|}{ Received DHM ${ }^{\mathrm{a}}$} \\
\hline Any DHM & $233(87.6)$ & $103(85.8)$ & $130(89.0)$ & 0.43 \\
\hline Only DHM & $23(8.7)$ & $10(8.3)$ & $13(8.9)$ & 0.87 \\
\hline \multicolumn{5}{|l|}{ Respiratory support ${ }^{\mathrm{a}}$} \\
\hline Oxygen & $228(85.7)$ & $101(84.2)$ & $127(87.0)$ & 0.51 \\
\hline Nasal CPAP & $213(80.1)$ & $91(75.8)$ & $122(83.6)$ & 0.12 \\
\hline Ventilation & $185(69.6)$ & $84(70.0)$ & $101(69.2)$ & 0.88 \\
\hline CVL days ${ }^{c}$ & $9[7,13]$ & $12[9,17]$ & $7[5,9]$ & $<0.0001$ \\
\hline PN days ${ }^{c}$ & $8[6,12]$ & $11[8,15]$ & $6[5,8]$ & $<0.0001$ \\
\hline 28-day GV (g/kg/day) ${ }^{\mathrm{b}}$ & $11.6(3.5)$ & $11.1(3.8)$ & $11.9(3.2)$ & 0.059 \\
\hline Days to return to birth weight ${ }^{b}$ & $8.8(3.7)$ & $8.7(3.6)$ & $8.9(3.7)$ & 0.71 \\
\hline $\begin{array}{l}\text { Change in z-score birth to } \\
28 \text { days }^{\text {b }}\end{array}$ & $-0.8(0.5)$ & $-0.83(0.5)$ & $-0.77(0.5)$ & 0.28 \\
\hline $\mathrm{NEC}^{\mathrm{a}}$ & $23(8.7)$ & $11(9.2)$ & $12(8.2)$ & 0.78 \\
\hline Culture-proven sepsis ${ }^{\mathrm{a}}$ & $26(9.8)$ & $16(13.3)$ & $10(6.9)$ & 0.08 \\
\hline $\mathrm{BPD}^{\mathrm{a}}$ & $161(52.3)$ & $59(49.2)$ & $80(54.8)$ & 0.36 \\
\hline
\end{tabular}

$N$ number, $S G A$ small-for-gestational age at birth, $G A$ gestational age, $D H M$ donor human milk, $C P A P$ continuous positive airway pressure, $C V L$ central venous line, $P N$ parenteral nutrition, $G V$ growth velocity, $N E C$ necrotizing enterocolitis, $B P D$ bronchopulmonary dysplasia.

${ }^{*} p$ value from Chi Square tests, Fisher's Exact tests, Student's $t$ tests and Wilcoxon Rank Sum tests as appropriate.

a Superscript identify the descriptive statistics which is "a" for frequency (percent).

buperscript identify the descriptive statistics which is " $b$ " for mean (standard deviation).

"Superscript identify the descriptive statistics which is "c" for median [interquartile range]. $2(\beta=-0.46, \mathrm{SE}=0.07$ and $p$ value $<0.0001)$. Similarly, the analysis for the number of PN days was also significantly lower in epoch $2(\beta=-0.45, \mathrm{SE}=0.05$ and $p$ value $<0.0001)$. Since growth velocity was significantly different between epochs in adjusted analysis, similar regression analysis including SGA status to replace birth weight, was performed to identify whether weight $z$-score change from birth to 28 postnatal days differed significantly by epoch when controlling for the other factors. It did not $(p=0.12)$.

In comparison of morbidities between groups, no infants in this study experienced spontaneous intestinal perforation. No difference was observed between epochs in BPD, NEC, or late onset-sepsis, except the proportion of infants born <
$1 \mathrm{~kg}$ who developed NEC was significantly lower in Epoch 2. This difference likely is not related to the revised feeding protocol and, instead, demonstrates the natural variation in NEC incidence at a single institution over a 24-month period.

\section{Discussion}

After implementing an evidence based revised feeding guideline which included discontinuing the practice of gastric residual monitoring, decreasing number of days of trophic feeding and progressive enteral feeding advancement, $83 \%$ of infants $1-1.5 \mathrm{~kg}$ and $77 \%$ of infants $<1 \mathrm{~kg}$ 
Table 2 Baseline demographic and outcomes by birth weight groups compared between epochs.

\begin{tabular}{|c|c|c|c|c|c|c|}
\hline \multirow[t]{2}{*}{ Descriptor } & \multicolumn{2}{|c|}{ Birth weight $<1 \mathrm{~kg}$} & \multirow[b]{2}{*}{$p$ value* } & \multicolumn{2}{|c|}{ Birth weight $1-1.5 \mathrm{~kg}$} & \multirow[b]{2}{*}{$p$ value* } \\
\hline & $\begin{array}{l}\text { Epoch } 1 \\
N=55\end{array}$ & $\begin{array}{l}\text { Epoch } 2 \\
N=58\end{array}$ & & $\begin{array}{l}\text { Epoch } 1 \\
N=68\end{array}$ & $\begin{array}{l}\text { Epoch } 2 \\
N=89\end{array}$ & \\
\hline Female $^{\mathrm{a}}$ & $35(63.6)$ & $30(51.7)$ & 0.20 & $33(48.5)$ & $45(50.6)$ & 0.80 \\
\hline Race/ethnicity $^{\mathrm{a}}$ & & & 0.36 & & & 0.79 \\
\hline Non-Hispanic black & $28(52.8)$ & $38(65.5)$ & & $38(56.7)$ & $44(50.0)$ & \\
\hline Non-Hispanic white & $22(41.5)$ & $18(31.0)$ & & $25(37.3)$ & $40(45.5)$ & \\
\hline Hispanic & $2(3.8)$ & $2(3.5)$ & & $3(4.5)$ & $3(3.4)$ & \\
\hline Other & $1(1.9)$ & 0 & & $1(1.5)$ & $1(1.1)$ & \\
\hline Antenatal steroids ${ }^{\mathrm{a}}$ & $45(84.9)$ & $49(84.5)$ & 0.95 & $59(88.1)$ & $80(90.9)$ & 0.56 \\
\hline $\mathrm{SGA}^{\mathrm{a}}$ & $4(7.6)$ & $7(12.1)$ & 0.43 & $6(9.0)$ & $3(3.4)$ & 0.18 \\
\hline Birth weight $(\mathrm{kg})^{\mathrm{b}}$ & $0.75(0.2)$ & $0.77(0.1)$ & 0.56 & $1.2(0.1)$ & $1.2(0.2)$ & 0.97 \\
\hline Birth GA (weeks) ${ }^{\mathrm{b}}$ & $26.0(1.9)$ & $26.2(1.7)$ & 0.52 & $29.8(1.8)$ & $29.4(1.5)$ & 0.22 \\
\hline $\begin{array}{l}\text { First feed (postnatal } \\
\text { hours) }\end{array}$ & $12[9,33]$ & $9.5[8,27]$ & 0.20 & $8[6,11]$ & $8[7,10]$ & 0.75 \\
\hline \multicolumn{7}{|l|}{ Received $\mathrm{DHM}^{\mathrm{a}}$} \\
\hline Any DHM & $43(81.1)$ & $51(87.9)$ & 0.32 & $60(89.6)$ & $79(89.8)$ & 0.96 \\
\hline Only DHM & $2(3.8)$ & $7(12.1)$ & 0.11 & $8(11.9)$ & $6(6.8)$ & 0.27 \\
\hline \multicolumn{7}{|l|}{ Respiratory support ${ }^{\mathrm{a}}$} \\
\hline Oxygen & $53(100)$ & $55(94.8)$ & 0.09 & 48 (71.6) & $72(81.8)$ & 0.13 \\
\hline Nasal CPAP & $34(64.2)$ & $38(65.5)$ & 0.88 & $57(85.1)$ & $84(95.5)$ & 0.04 \\
\hline Ventilation & $49(92.5)$ & $51(87.9)$ & 0.43 & $35(52.2)$ & $50(56.8)$ & 0.57 \\
\hline CVL days ${ }^{\mathrm{c}}$ & $15[13,23]$ & $9[7,10]$ & $<0.0001$ & $9[8,10]$ & $5[5,6]$ & $<0.0001$ \\
\hline PN days ${ }^{\mathrm{c}}$ & $16[13,23]$ & $8[8,12]$ & $<0.0001$ & $8[6,9]$ & $5[5,6]$ & $<0.0001$ \\
\hline 28-day GV (g/kg/day) ${ }^{\mathrm{b}}$ & $10.8(4.7)$ & $12.0(3.6)$ & 0.13 & $11.4(2.8)$ & $11.8(2.8)$ & 0.30 \\
\hline $\begin{array}{l}\text { Days to return to birth } \\
\text { weight }\end{array}$ & $8.8(3.7)$ & $8.3(3.6)$ & 0.46 & $8.6(3.4)$ & $9.2(3.8)$ & 0.28 \\
\hline $\begin{array}{l}\text { Change in z-score birth to } \\
28 \text { days }{ }^{b}\end{array}$ & $-0.8(0.6)$ & $-0.7(0.5)$ & 0.40 & $-0.9(0.4)$ & $-0.8(0.4)$ & 0.39 \\
\hline $\mathrm{NEC}^{\mathrm{a}}$ & $7(13.2)$ & $1(1.7)$ & 0.02 & $4(6.0)$ & $11(12.5)$ & 0.17 \\
\hline Culture-proven sepsis ${ }^{\mathrm{a}}$ & $10(18.9)$ & $7(12.1)$ & 0.32 & $6(9.0)$ & $3(3.4)$ & 0.18 \\
\hline $\mathrm{BPD}^{\mathrm{a}}$ & $45(84.9)$ & $49(84.5)$ & 0.95 & $14(20.9)$ & $31(35.2)$ & 0.05 \\
\hline
\end{tabular}

$N$ number, $S G A$ small-for-gestational age at birth, $G A$ gestational age, DHM donor human milk, CPAP continuous positive airway pressure, $C V L$ central venous line, $P N$ parenteral nutrition, $G V$ growth velocity, $N E C$ necrotizing enterocolitis, $B P D$ bronchopulmonary dysplasia.

${ }^{*} p$ value from Chi Square tests, Fisher's Exact tests, Student's $t$ tests and Wilcoxon Rank Sum tests as appropriate.

a Superscript identify the descriptive statistics which is "a" for frequency (percent).

buperscript identify the descriptive statistics which is "b" for mean (standard deviation).

"Superscript identify the descriptive statistics which is "c" for median [interquartile range]. reached full feeds by 7 and 14 postnatal days, respectively. This change was associated with statistically and clinically significantly less days of CVL and PN in both unadjusted and adjusted comparisons. In addition, in adjusted models, mean 28-day growth velocity was significantly higher for the epoch receiving the revised feeding protocol (Epoch 2). However, weight $z$-score change from birth to 28-days was not significantly different between epochs in either univariate or multivariate analysis. This study focused specifically on how the revision of the feeding protocol, as compared by epochs, related to the outcomes of interest. Investigation of how other parameters such as GA, SGA status, and antenatal steroids was not performed in this study but may be of interest in future research of VLBW infant feeding.

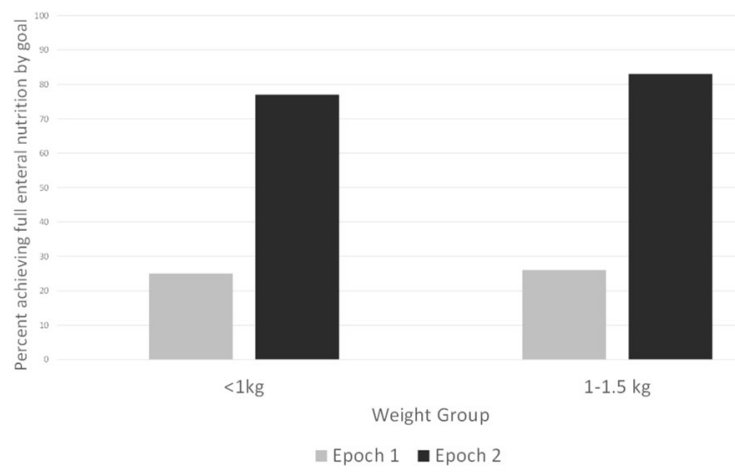

Fig. 2 Percent of infants attaining full enteral nutrition by 2 weeks (birthweight $<1 \mathrm{~kg}$ ) and 1 week (birthweight $1-1.5 \mathrm{~kg}$ ) between epochs. Analysis by Chi-Square test. 

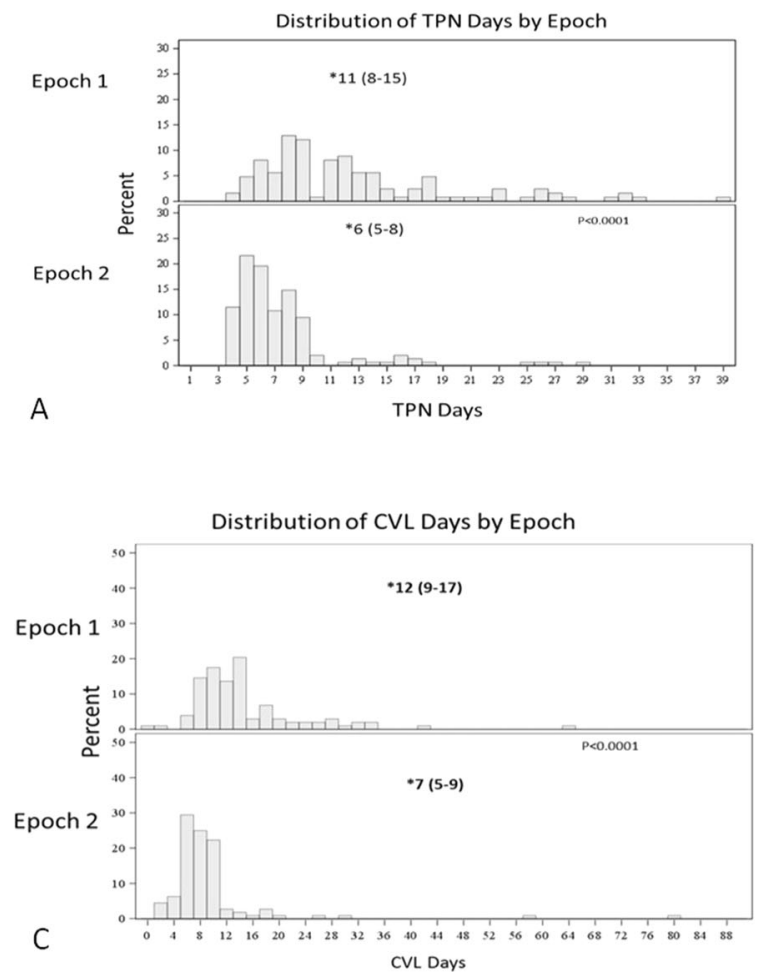

Fig. 3 Duration of parenteral nutrition and central lines compared between epochs. Histograms comparing the distribution between infants in Epoch 1 and Epoch 2 for parenteral nutrition (TPN) days (a) and the same comparison of TPN days by birthweight categories (b). Histograms comparing the distribution of central venous line (CVL)

The revised feeding protocol had multiple components based on evidence review. The revisions included a discontinuation of routine gastric residual monitoring which was based on recent evidence demonstrating no utility of this practice and the potential that it is associated with a delay in achievement of full enteral nutrition [15]. Recently, results of a randomized, controlled trial verified the results of earlier observational studies and showed increased enteral nutrition delivery and improved weight gain when gastric residuals were not routinely monitored [16]. The revised guidelines in our study also included a shortened duration of trophic feeds to 1 day for infants $1-1.5 \mathrm{~kg}$ and to 3 days for infants $<1 \mathrm{~kg}$. A previous retrospective cohort study demonstrated 3 days of trophic feeds was associated with faster achievement of full enteral nutrition in extremely preterm infants [17]. In our study, the increased daily feed advancement volume to $25-30 \mathrm{ml} / \mathrm{kg} /$ day was based on published systematic review of the evidence [7]. More recently, the results of 2-year outcomes of a multicenter randomized trial comparing $30 \mathrm{~mL} / \mathrm{kg} /$ day with $18 \mathrm{~mL} / \mathrm{kg}$ / day were published [9]. In this recent publication, a more rapid advancement of volume was associated with shorter duration to achieve full feeds, but no significant difference in 2-year outcomes was observed except for infants receiving formula-only feeds who demonstrated better
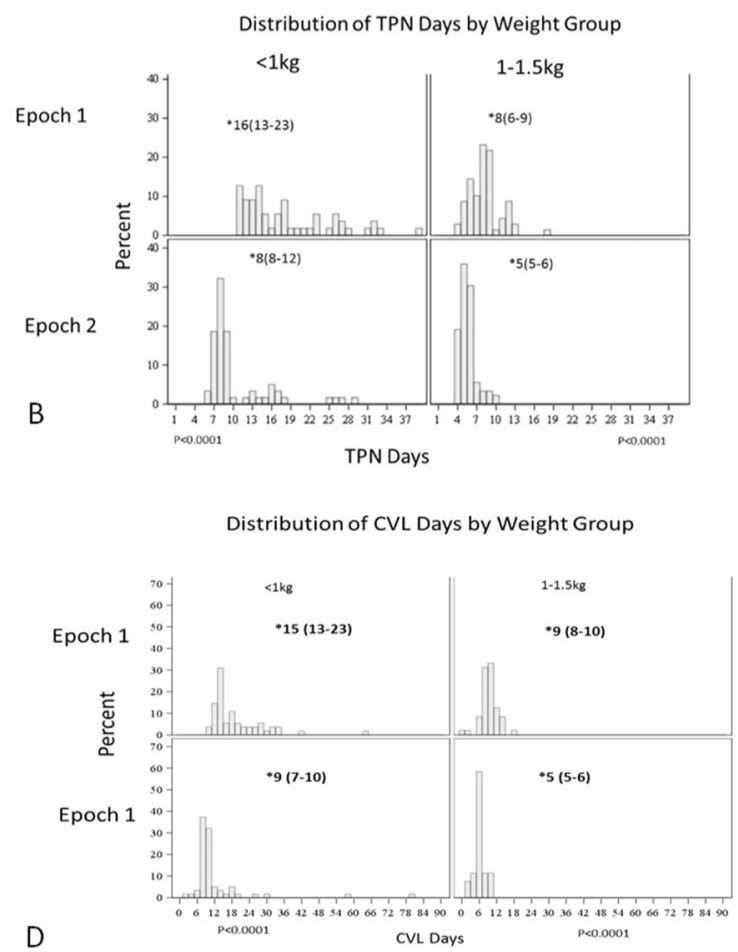

days between infants in Epoch 1 and Epoch 2 (c) and the same comparison of CVL days by birthweight categories (d). *Median (interquartile range) given for each distribution. Statistical comparison by Wilcoxon Rank Sum Tests.

survival without moderate or severe neurodevelopmental disability in the slower feed volume advancement group. Of note, the infants in this multi-center randomized trial did not start feeds on the first postnatal day [9].

In this study with human milk feeds initiated on the first postnatal day, full feeds were achieved with lower CVL and PN days and higher growth velocity when adjusted for potential confounders. Other retrospective cohort studies have demonstrated similar results but occurred in an older patient population [18], included parenteral nutrition revisions [19], or compared a decrease in the number of days prior to feed progression. A decrease in the number of days prior to feed progression was associated with improved 28-day GV [20]. A similar significant increase in GV was found in our study when adjusted for potential confounders. In addition in our results, the change in $z$-score for both epochs were similar to the change observed by Rochow et al., though they measured change in $z$-scores from birth to 21 postnatal days, instead of 28 days, and included infants 25-34 weeks' GA [21]. In our study, no difference in the number of days to return to birth weight was observed between epochs which may reflect the fact that PN practices did not differ between groups [22].

The strengths of this study include [1] the similarity of infants in both epochs in terms of sex, birthweight, and GA, 
and [2] the inclusion of infants who were SGA in these standard feeding protocols. Days of exposure to PN were decreased in this study hence potentially decreasing the risks and costs associated with PN. Days with a CVL were decreased hence decreasing the days on which line complications could occur as well as the risk for central line-associated blood stream infection.

Limitations of this study include that not every potential complication of a revised feeding potential was studied. For example, the number of abdominal radiographs to evaluate potential feeding intolerance was not measured. In addition, although the study center is a tertiary regional center covering an eight-county region of South Carolina, this was still a single-center cohort study. GV was only measured at 28 postnatal days. Therefore, the effect on long-term growth is not known.

\section{Conclusion}

Despite clinical concern that "pushing" infants to feed earlier and more quickly would lead to more stops and starts of feeding and therefore longer time to full enteral feeds, this study shows that achieving full enteral nutrition within 1 postnatal week for infants born $1-1.5 \mathrm{~kg}$ and within 2 postnatal weeks for infants born $<1 \mathrm{~kg}$ is feasible and can be applied to clinical practice. The magnitude of the decrease in central line and parenteral nutrition demonstrates the potential for decreased patient morbidity and hospital cost associated with this strategy.

Acknowledgements The authors acknowledge Dr. Julie Ross for her leadership in quality improvement initiatives in the neonatal intensive care unit, the entire Medical University of South Carolina Milky Weigh nutrition quality improvement team, and the physicians, nurses, and dietitians who follow these evidence-based protocols in their care of VLBW infants. In addition, the authors acknowledge Dr. Martina Mueller who assisted in the statistical methodology. The authors have no disclosures or sources of any support for this work such as grants and/or equipment and drugs.

Author contributions AF and SNT provided substantial contributions to the conception and design of the work. AF performed data acquisition. All authors made substantial contributions in data analysis, data interpretation, and drafting the work with final approval of the version to be published.

\section{Compliance with ethical standards}

Conflict of interest SNT has received research funding from NIH and the Allen Foundation. SNT has served as a consultant for Alcresta Therapeutics and serves as a volunteer member of the Mother's Milk Bank Northeast medical advisory board. AF and JCN have no potential conflict of interest to declare.

Publisher's note Springer Nature remains neutral with regard to jurisdictional claims in published maps and institutional affiliations.

\section{References}

1. Embleton NE, Pang N, Cooke RJ. Postnatal malnutrition and growth retardation: an inevitable consequence of current recommendations in preterm infants? Pediatrics. 2001;107:270-3.

2. Ehrenkranz RA, Dusick AM, Vohr BR, Wright LL, Wrage LA, Poole WK. Growth in the neonatal intensive care unit influences neurodevelopmental and growth outcomes of extremely low birth weight infants. Pediatrics. 2006;117:1253-61.

3. Dusick AM, Poindexter BB, Ehrenkranz RA, Lemons JA. Growth failure in the preterm infant: can we catch up? Semin Perinatol. 2003;27:302-10.

4. Henderson G, Craig S, Brocklehurst P, McGuire W. Enteral feeding regimens and necrotising enterocolitis in preterm infants: a multicentre case-control study. Arch Dis Child Fetal Neonatal Ed. 2009;94:F120-3.

5. McKeown RE, Marsh TD, Amarnath U, Garrison CZ, Addy CL, Thompson SJ, et al. Role of delayed feeding and of feeding increments in necrotizing enterocolitis. J Pediatrics. 1992;121(5 Pt 1):764-70.

6. Berseth CL, Bisquera JA, Paje VU. Prolonging small feeding volumes early in life decreases the incidence of necrotizing enterocolitis in very low birth weight infants. Pediatrics. 2003; 111:529-34.

7. Oddie SJ, Young L, McGuire W. Slow advancement of enteral feed volumes to prevent necrotising enterocolitis in very low birth weight infants. Cochrane database Syst Rev. 2017;8:CD001241.

8. Morgan J, Young L, McGuire W. Slow advancement of enteral feed volumes to prevent necrotising enterocolitis in very low birth weight infants. Cochrane Datab System Rev. 2015:Cd001241. https://doi.org/10.1002/14651858.CD001241.pub6.

9. Dorling J, Abbott J, Berrington J, Bosiak B, Bowler U, Boyle E, et al. Controlled trial of two incremental milk-feeding rates in preterm infants. N Engl J Med. 2019;381:1434-43.

10. Dutta S, Singh B, Chessell L, Wilson J, Janes M, McDonald K, et al. Guidelines for feeding very low birth weight infants. Nutrients. 2015;7:423-42.

11. Hamilton E, Massey C, Ross J, Taylor S. Early enteral feeding in very low birth weight infants. Early Hum Dev. 2014;90:227-30.

12. Alshaikh B, Dharel D, Yusuf K, Singhal N. Early total enteral feeding in stable preterm infants: a systematic review and metaanalysis. J Matern-Fetal Neonatal Med. 2019:CD001241. https:// doi.org/10.1080/14767058.2019.1637848.

13. Fenton TR, Kim JH. A systematic review and meta-analysis to revise the Fenton growth chart for preterm infants. BMC Pediatr. 2013;13:59.

14. Fenton TR, Anderson D, Groh-Wargo S, Hoyos A, Ehrenkranz RA, Senterre T. An attempt to standardize the calculation of growth velocity of preterm infants-evaluation of practical bedside methods. J Pediatr. 2018;196:77-83.

15. Torrazza RM, Parker LA, Li Y, Talaga E, Shuster J, Neu J. The value of routine evaluation of gastric residuals in very low birth weight infants. J Perinatol. 2015;35:57-60.

16. Parker LA, Weaver M, Murgas Torrazza RJ, Shuster J, Li N, Krueger $\mathrm{C}$, et al. Effect of gastric residual evaluation on enteral intake in extremely preterm infants: a randomized clinical trial. JAMA Pediatr. 2019;173:534-43.

17. Salas AA, Kabani N, Travers CP, Phillips V, Ambalavanan N, Carlo WA. Short versus extended duration of trophic feeding to reduce time to achieve full enteral feeding in extremely preterm infants: an observational study. Neonatology. 2017;112:211-6.

18. Morton SU, Belfort MB, Kahlon PS, Hajizadeh Barfjani S, Rudie C, Hashim E, et al. Reducing time to initiation and advancement of enteral feeding in an all-referral neonatal intensive care unit. J Perinatol. 2018;38:936-43. 
19. Thoene MK, Lyden E, Anderson-Berry A. Improving nutrition outcomes for infants $<1500$ grams with a progressive, evidenced-based enteral feeding protocol. Nutr Clin Pract. 2018; 33:647-55.

20. Culpepper C, Hendrickson K, Marshall S, Benes J, Grover TR. Implementation of feeding guidelines hastens the time to initiation of enteral feeds and improves growth velocity in very low birthweight infants. Adv Neonatal Care. 2017;17:139-45.
21. Rochow N, Raja P, Liu K, Fenton T, Landau-Crangle E, Gottler S, et al. Physiological adjustment to postnatal growth trajectories in healthy preterm infants. Pediatr Res. 2016;79:870-9.

22. Perrem L, Semberova J, O'Sullivan A, Kieran EA, O'Donnell $\mathrm{CPF}$, White $\mathrm{MJ}$, et al. Effect of early parenteral nutrition discontinuation on time to regain birth weight in very low birth weight infants: a randomized controlled trial. J Parenter Enter Nutr. 2019;43:883-90. 